\title{
PERIODS OF HOMEOMORPHISMS ON CLOSED SURFACES
}

\author{
JUAN LUIS GARCÍA GUIRAO ${ }^{1}$ AND JAUME LLIBRE ${ }^{2}$
}

\begin{abstract}
The goal of this paper is to show what information on the set of periodic points of a homeomorphism on a closed surface can be obtained using the action of this homeomorphism on the homological groups of the closed surface.
\end{abstract}

\section{INTRODUCTION}

Here a closed surface means a connected compact surface with or without boundary, orientable or not. More precisely, an orientable connected compact surface without boundary of genus $g \geq 0, \mathbb{M}_{g}$, is homeomorphic to the sphere if $g=0$, to the torus if $g=1$, or to the connected sum of $g$ copies of the torus if $g \geq 2$. An orientable connected compact surface with boundary of genus $g \geq 0$, $\mathbb{M}_{g, b}$, is homeomorphic to $\mathbb{M}_{g}$ minus a finite number $b>0$ of open discs having pairwise disjoint closure. In what follows $\mathbb{M}_{g, 0}=\mathbb{M}_{g}$.

A non-orientable connected compact surface without boundary of genus $g \geq$ $1, \mathbb{N}_{g}$, is homeomorphic to the real projective plane if $g=1$, or to the connected sum of $g$ copies of the real projective plane if $g>1$. A non-orientable connected compact surface with boundary of genus $g \geq 1, \mathbb{N}_{g, b}$, is homeomorphic to $\mathbb{N}_{g}$ minus a finite number $b>0$ of open discs having pairwise disjoint closure. In what follows $\mathbb{N}_{g, 0}=\mathbb{N}_{g}$.

Let $f: \mathbb{X} \rightarrow \mathbb{X}$ be a homeomorphism on a closed surface $\mathbb{X}$. A point $x \in \mathbb{X}$ is periodic of period $n$ if $f^{n}(x)=x$ and $f^{k}(x) \neq x$ for $k=1, \ldots, n-1$. We denote by $\operatorname{Per}(f)$ the set of periods of all periodic points of $f$. The aim of the present paper is to provide some information on $\operatorname{Per}(f)$. The statement of our first result is.

Theorem 1. Let $\mathbb{X}$ be a closed surface and let $f$ be a self-homeomorphism into $\mathbb{X}$. If $\mathbb{X}=\mathbb{M}_{g, b}$, then the following statement hold.

(a) If $(g, b) \in\{(1,0),(0,2)\}$ (i.e. the torus and the closed annulus respectively), then there is no information on the set $\operatorname{Per}(f)$.

(b) If $(g, b)=(0,0)$ (i.e. the 2 -dimensional sphere), then $\operatorname{Per}(f) \cap\{1,2\} \neq$ $\emptyset$.

(c) If $(g, b)=(0,1)$ (i.e. the 2 -dimensional disc), then $1 \in \operatorname{Per}(f)$.

(d) If $g>1$ and $b=0$, then $\operatorname{Per}(f) \cap\{1,2, \ldots, 2 g\} \neq \emptyset$.

Key words and phrases. Closed surface, homeomorphism, Lefschetz fixed point theory. 2010 Mathematics Subject Classification: 58F20, 37C05, 37C25, 37C30. 
(e) If $g>1$ and $b>0$, then $\operatorname{Per}(f) \cap\{1,2, \ldots, 2 g+b-1\} \neq \emptyset$.

If $\mathbb{X}=\mathbb{N}_{g, b}$, then the following statements hold.

$(f)$ If $(g, b) \in\{(2,0),(1,1)\}$ (i.e. the Klein bottle and the Möebius band respectively), there is no information on the set $\operatorname{Per}(f)$.

(g) If $(g, b)=(1,0)$ (i.e. the projective plane), then $1 \in \operatorname{Per}(f)$.

(h) If $(g, b)$ does not satisfy the assumptions of statements $(f)$ or $(g)$, then $\operatorname{Per}(f) \cap\{1,2, \ldots, g+b-1\} \neq \emptyset$.

The proof of Theorem 1 is done in section 2. The main tool for proving it is a result due to Fuller [4].

The results of Theorem 1 restricted to the orientable closed surfaces without boundary, i.e. for the closed surfaces $\mathbb{M}_{g, 0}$, where already obtained by Franks and Llibre in [3].

The objective of the rest of the paper is to improve the information provided in Theorem 1 using as a main tool the Lefschetz fixed point theory. We shall follow the ideas of Franks and Llibre in [3] when they improve the results of Theorem 1 for the homeomorphisms of the closed surfaces $\mathbb{M}_{g, 0}$, see Theorems 5 and 6 .

Let $A$ be an $n \times n$ complex matrix. A $k \times k$ principal submatrix of $A$ is a submatrix lying in the same set of $k$ rows and columns, and a $k \times k$ principal minor is the determinant of such a principal submatrix. There are $\left(\begin{array}{l}n \\ k\end{array}\right)$ different $k \times k$ principal minors of $A$, and the sum of these is denoted by $E_{k}(A)$. In particular, $E_{1}(A)$ is the trace of $A$, and $E_{n}(A)$ is the determinant of $A$, denoted by $\operatorname{det}(A)$.

It is well known that the characteristic polynomial of $A$ is given by

$$
\operatorname{det}(t I-A)=t^{n}-E_{1}(A) t^{n-1}+E_{2}(A) t^{n-2}-\ldots+(-1)^{n} E_{n}(A) .
$$

Our main result is state in the following theorem.

Theorem 2. Let $f: \mathbb{X} \rightarrow \mathbb{X}$ be a homeomorphism and let $A$ be the integral matrix of the isomorphism $f_{* 1}: H_{1}(\mathbb{X}, \mathbb{Q}) \rightarrow H_{1}(\mathbb{X}, \mathbb{Q})$ induced by $f$ on the first homology group of $\mathbb{X}$. If $\mathbb{X}$ is either $\mathbb{M}_{g, b}$ with $b>0$, or $\mathbb{N}_{g, b}$ with $b \geq 0$, then the following statements hold.

(a) If $E_{1}(A) \neq 1$, then $1 \in \operatorname{Per}(f)$.

(b) If $E_{1}(A)=1$ and $E_{2}(A) \neq 0$, then $\operatorname{Per}(f) \cap\{1,2\} \neq \emptyset$.

If $\mathbb{X}=\mathbb{M}_{g, b}$ with $b>0$, then the following statement hold.

(c) If $2 g+b-1 \geq 3, E_{1}(A)=1, E_{2}(A)=0$ and $k$ is the smallest integer of the set $\{3,4, \ldots, 2 g+b-1\}$ such that $E_{k}(A) \neq 0$, then $\operatorname{Per}(f)$ has a periodic point of period a divisor of $k$.

If $\mathbb{X}=\mathbb{N}_{g, b}$ with $b \geq 0$, then the following statement hold.

(d) If $g+b-1 \geq 3, E_{1}(A)=1, E_{2}(A)=0$ and $k$ is the smallest integer of the set $\{3,4, \ldots, g+b-1\}$ such that $E_{k}(A) \neq 0$, then $\operatorname{Per}(f)$ has a periodic point of period a divisor of $k$.

Theorem 2 is proven in section 3 . 


\section{Proof of Theorem 1}

Let $f$ be continuous self-map defined on $\mathbb{M}_{g, b}$ or $\mathbb{N}_{g, b}$, respectively. For a closed surface, the homological groups with coefficients in $\mathbb{Q}$ are linear vector spaces over $\mathbb{Q}$. We recall the homological spaces of $\mathbb{M}_{g, b}$ with coefficients in $\mathbb{Q}$, i.e.

$$
H_{k}\left(\mathbb{M}_{g, b}, \mathbb{Q}\right)=\mathbb{Q} \oplus n^{n_{k}} \oplus \mathbb{Q},
$$

where $n_{0}=1, n_{1}=2 g$ if $b=0, n_{1}=2 g+b-1$ if $b>0, n_{2}=1$ if $b=0$, and $n_{2}=0$ if $b>0$; and the induced linear maps $f_{* k}: H_{k}\left(\mathbb{M}_{g, b}, \mathbb{Q}\right) \rightarrow H_{k}\left(\mathbb{M}_{g, b}, \mathbb{Q}\right)$ by $f$ on the homological group $H_{k}\left(\mathbb{M}_{g, b}, \mathbb{Q}\right)$ are $f_{* 0}=(1), f_{* 2}=(d)$ where $d$ is the degree of the map $f$ if $b=0, f_{* 2}=0$ if $b>0$, and $f_{* 1}=A$ where $A$ is an $n_{1} \times n_{1}$ integral matrix (see for additional details [6, 7]).

We recall that the homological groups of $\mathbb{N}_{g, b}$ with coefficients in $\mathbb{Q}$, i.e.

$$
H_{k}\left(\mathbb{N}_{g, b}, \mathbb{Q}\right)=\mathbb{Q} \oplus n^{n} \cdot \oplus \mathbb{Q},
$$

where $n_{0}=1, n_{1}=g+b-1$ and $n_{2}=0$; and the induced linear maps are $f_{* 0}=(1)$ and $f_{* 1}=A$ where $A$ is an $n_{1} \times n_{1}$ integral matrix (see again for additional details $[6,7])$.

The proof of Theorem 1 is a consequence of a general result from polyhedron homeomorphisms proved in [4], see also Halpern [5] and Brown [1] for more details on it.

Theorem 3 (Fuller's Theorem). Let $f$ be a homeomorphism of a compact polyhedron $X$ into itself. If the Euler characteristic of $X$ is not zero, then $f$ has a periodic point with period not greater that the maximum of $\sum_{k \text { odd }} B_{k}(X)$ and $\sum_{k \text { even }} B_{k}(X)$, where $B_{k}(X)$ denotes the $k$-th Betti number of $X$.

Proof of Theorem 1. Assume $\mathbb{X}=\mathbb{M}_{g, b}$. Since for a closed surface $\mathbb{M}_{g, b}$ its homological groups with rational coefficients are $H_{0}\left(\mathbb{M}_{g, b}, \mathbb{Q}\right)=\mathbb{Q}, H_{1}\left(\mathbb{M}_{g, b}, \mathbb{Q}\right)=$ $\mathbb{Q} \oplus \cdot 2 g . \oplus \mathbb{Q}$ and $H_{2}\left(\mathbb{M}_{g, b}, \mathbb{Q}\right)=\mathbb{Q}$ if $b=0$, and $H_{0}\left(\mathbb{M}_{g, b}, \mathbb{Q}\right)=\mathbb{Q}, H_{1}\left(\mathbb{M}_{g, b}, \mathbb{Q}\right)=$ $\mathbb{Q} \oplus{ }^{2 g+{ }^{\prime}-1} \oplus \mathbb{Q}$ and $H_{2}\left(\mathbb{M}_{g, b}, \mathbb{Q}\right)=0$ if $b>0$, then its Euler characteristic $\mathcal{X}\left(\mathbb{M}_{g, b}\right)=B_{0}\left(\mathbb{M}_{g, b}\right)-B_{1}\left(\mathbb{M}_{g, b}\right)+B_{2}\left(\mathbb{M}_{g, b}\right)$ is equal to $2-2 g$ if $b=0$, and $2-2 g-b$ if $b>0$, where $B_{k}\left(\mathbb{M}_{g, b}\right)=\operatorname{dim}_{\mathbb{Q}}\left(H_{k}\left(\mathbb{M}_{g, b}, \mathbb{Q}\right)\right)$.

Since

$$
\begin{aligned}
\sum_{k \text { even }} B_{k}\left(\mathbb{M}_{g, b}\right)=2 \neq 0 \text { and } \sum_{k \text { odd }} B_{k}\left(\mathbb{M}_{g, b}\right)=2 g & \text { if } b=0, \\
\sum_{k \text { even }} B_{k}\left(\mathbb{M}_{g, b}\right)=1 \neq 0 \text { and } \sum_{k \text { odd }} B_{k}\left(\mathbb{M}_{g, b}\right)=2 g+b-1 & \text { if } b>0,
\end{aligned}
$$

the orientable closed surfaces for which the Fuller's Theorem does not provide any information on the set of periods $\operatorname{Per}(f)$ are the ones having zero Euler characteristic, i.e. when $g=1$ and $b=0$, and $g=0$ and $b=2$. Therefore statement (a) is proved.

If $(g, b)=(0,0)$ then $\max \left\{\sum_{k \text { even }} B_{k}\left(\mathbb{M}_{g, b}\right)=2, \sum_{k \text { odd }} B_{k}\left(\mathbb{M}_{g, b}\right)=0\right\}=2$, then by Theorem 3 it follows that $\operatorname{Per}(f) \cap\{1,2\} \neq \emptyset$. Hence statement (b) follows. 
If $(g, b)=(0,1)$ then $\max \left\{\sum_{k \text { even }} B_{k}\left(\mathbb{M}_{g, b}\right)=1, \sum_{k \text { odd }} B_{k}\left(\mathbb{M}_{g, b}\right)=0\right\}=1$, then by Theorem 3 it follows that $1 \in \operatorname{Per}(f)$. So statement (c) follows.

If $g>1$ and $b=0$ then $\max \left\{\sum_{k \text { even }} B_{k}\left(\mathbb{M}_{g, b}\right)=2, \sum_{k \text { odd }} B_{k}\left(\mathbb{M}_{g, b}\right)=\right.$ $2 g\}=2 g$, then by Theorem 3 it follows that $\operatorname{Per}(f) \cap\{1,2, \ldots, 2 g\} \neq \emptyset$. Hence statement $(\mathrm{d})$ is proved.

If $g>1$ and $b>0$ then $\max \left\{\sum_{k \text { even }} B_{k}\left(\mathbb{M}_{g, b}\right)=1, \sum_{k \text { odd }} B_{k}\left(\mathbb{M}_{g, b}\right)=\right.$ $2 g\}=2 g+b-1$, then by Theorem 3 it follows that $\operatorname{Per}(f) \cap\{1,2, \ldots, 2 g+b-1\} \neq$ $\emptyset$. Therefore statement (e) follows.

Assume $\mathbb{X}=\mathbb{N}_{g, b}$. Since for a closed surface $\mathbb{N}_{g, b}$ its homological groups with rational coefficients are $H_{0}\left(\mathbb{M}_{g, b}, \mathbb{Q}\right)=\mathbb{Q}, H_{1}\left(\mathbb{M}_{g, b}, \mathbb{Q}\right)=\mathbb{Q} \oplus{ }^{g+b-1} \oplus \mathbb{Q}$ and $H_{2}\left(\mathbb{M}_{g, b}, \mathbb{Q}\right)=0$, then its Euler characteristic $\mathcal{X}\left(\mathbb{N}_{g, b}\right)=B_{0}\left(\mathbb{N}_{g, b}\right)-B_{1}\left(\mathbb{N}_{g, b}\right)+$ $B_{2}\left(\mathbb{N}_{g, b}\right)=2-g-b$, where $B_{k}\left(\mathbb{N}_{g, b}\right)=\operatorname{dim}_{\mathbb{Q}}\left(H_{k}\left(\mathbb{N}_{g, b}, \mathbb{Q}\right)\right)$.

Since

$$
\sum_{k \text { even }} B_{k}\left(\mathbb{M}_{g, b}\right)=1 \neq 0 \text { and } \sum_{k \text { odd }} B_{k}\left(\mathbb{M}_{g, b}\right)=g+b-1,
$$

the non-orientable closed surfaces for which the Fuller's Theorem does not provide any information on the set of periods $\operatorname{Per}(f)$ are the ones having zero Euler characteristic, i.e. when $g=2$ and $b=0$, and $g=1$ and $b=1$. Therefore statement ( $\mathrm{f})$ is proved.

If $(g, b)=(1,0)$ then $\max \left\{\sum_{k \text { even }} B_{k}\left(\mathbb{M}_{g, b}\right)=1, \sum_{k \text { odd }} B_{k}\left(\mathbb{M}_{g, b}\right)=0\right\}=1$, then by Theorem 3 it follows that $1 \in \operatorname{Per}(f)$. So statement (g) follows.

If $(g, b)$ does not satisfy the assumptions of statements $(f)$ or $(g)$, then $\max \left\{\sum_{k \text { even }} B_{k}\left(\mathbb{M}_{g, b}\right)=1, \sum_{k \text { odd }} B_{k}\left(\mathbb{M}_{g, b}\right)=g+b-1\right\}=g+b-1$, then by Theorem 3 it follows that $\operatorname{Per}(f) \cap\{1,2, \ldots, g+b-1\} \neq \emptyset$. Hence statement (h) is proved.

\section{Proof of Theorems 2}

Let $f: \mathbb{X} \rightarrow \mathbb{X}$ be a continuous map and let $\mathbb{X}$ be either $\mathbb{M}_{g, b}$ or $\mathbb{N}_{g, b}$. Then the Lefschetz number of $f$ is defined by

$$
L(f)=\operatorname{trace}\left(f_{* 0}\right)-\operatorname{trace}\left(f_{* 1}\right)+\operatorname{trace}\left(f_{* 2}\right) .
$$

For continuous maps and in particular for homeomorphisms $f$ defined on $\mathbb{X}$ the Lefschetz fixed point theorem states (see for instance [1]).

Theorem 4. If $L(f) \neq 0$ then $f$ has a fixed point.

With the aim of studying the periodic points of $f$ we shall use the Lefschetz numbers of the iterates of $f$, i.e. $L\left(f^{n}\right)$. Note that if $L\left(f^{n}\right) \neq 0$ then $f^{n}$ has a fixed point, and consequently $f$ has a periodic point of period a divisor of $n$. In order to study the whole sequence $\left\{L\left(f^{n}\right)\right\}_{n \geq 1}$ it is defined the formal Lefschetz zeta function of $f$ as

$$
Z_{f}(t)=\exp \left(\sum_{n=1}^{\infty} \frac{L\left(f^{n}\right)}{n} t^{n}\right) .
$$


The Lefschetz zeta function is in fact a generating function for the sequence of Lefschetz numbers $n$. In order to study the whole sequence $\left\{L\left(f^{n}\right)\right\}_{n \geq 1}$.

For a continuous self-map of a closed surface the Lefschetz zeta function is the rational function

$$
Z_{f}(t)=\frac{\operatorname{det}\left(I-t f_{* 1}\right)}{\operatorname{det}\left(I-t f_{* 0}\right) \operatorname{det}\left(I-t f_{* 2}\right)},
$$

see for more details Franks [2]. Then, for an orientation-preserving homeomorphism $f: \mathbb{M}_{g, b} \rightarrow \mathbb{M}_{g, b}$ we have

$$
Z_{f}(t)= \begin{cases}\frac{\operatorname{det}(I-t A)}{(1-t)^{2}} & \text { if } b=0 . \\ \frac{\operatorname{det}(I-t A)}{1-t} & \text { if } b>0,\end{cases}
$$

where $f_{* 1}=A$. Note that $f_{* 2}=(1)$ if $b=0$, and $f_{* 2}=(0)$ if $b>0$.

For an orientation-reversing homeomorphism $f: \mathbb{M}_{g, b} \rightarrow \mathbb{M}_{g, b}$ we have

$$
Z_{f}(t)= \begin{cases}\frac{\operatorname{det}(I-t A)}{1-t^{2}} & \text { if } b=0 . \\ \frac{\operatorname{det}(I-t A)}{1-t} & \text { if } b>0 .\end{cases}
$$

Note that $f_{* 2}=(-1)$ if $b=0$, and $f_{* 2}=(0)$ if $b>0$.

Finally, for a homeomorphism $f: \mathbb{N}_{g, b} \rightarrow \mathbb{N}_{g, b}$ we have

$$
Z_{f}(t)=\frac{\operatorname{det}(I-t A)}{1-t} .
$$

Using the Lefschetz zeta function the orientation preserving and reversing homeomorphisms on $\mathbb{M}_{g, 0}$ were studied in [3]. The results there obtained are the following two theorems. Here we shall study the homeomorphisms on $\mathbb{M}_{g, b}$ with $b>0$ and on $\mathbb{N}_{g, b}$ with $b \geq 0$.

Theorem 5 (Theorem 4 of [3]). Let $f: \mathbb{M}_{g, 0} \rightarrow \mathbb{M}_{g, 0}$ be an orientationpreserving homeomorphism and let $A$ be the $2 g \times 2 g$ integral matrix of the isomorphism $f_{* 1}: H_{1}\left(\mathbb{M}_{g, 0}, \mathbb{Q}\right) \rightarrow H_{1}\left(\mathbb{M}_{g, 0}, \mathbb{Q}\right)$ induced by $f$ on the first homology group of $\mathbb{M}_{g, 0}$. Then the following statements hold.

(a) If $g=0$, then $1 \in \operatorname{Per}(f)$.

(b) If $g>0$ and $E_{1}(A) \neq 2$, then $1 \in \operatorname{Per}(f)$.

(c) If $g>0, E_{1}(A)=2$ and $E_{2}(A) \neq 1$, then $\operatorname{Per}(f) \cap\{1,2\} \neq \emptyset$.

(d) If $g=1, E_{1}(A)=2$ and $E_{2}(A)=1$, then there is no information on $\operatorname{Per}(f)$.

(e) If $g>1, E_{1}(A)=2, E_{2}(A)=1$ and $k$ is the smallest integer of the set $\{3,4, \ldots, 2 g\}$ such that $E_{k}(A) \neq 0$, then $f$ has a periodic point of period a divisor of $k$. 
Theorem 6 (Theorem 3 of [3]). Let $f: \mathbb{M}_{g, 0} \rightarrow \mathbb{M}_{g, 0}$ be an orientationreversing homeomorphism and let $A$ be the $2 g \times 2 g$ integral matrix of the isomorphism $f_{* 1}: H_{1}\left(\mathbb{M}_{g, 0}, \mathbb{Q}\right) \rightarrow H_{1}\left(\mathbb{M}_{g, 0}, \mathbb{Q}\right)$ induced by $f$ on the first homology group of $\mathbb{M}_{g, 0}$. Then the following statements hold.

(a) If $g=0$, then $\operatorname{Per}(f) \cap\{1,2\} \neq \emptyset$.

(b) If $g>0$ and $E_{1}(A) \neq 0$, then $1 \in \operatorname{Per}(f)$.

(c) If $g>0, E_{1}(A)=0$ and $E_{2}(A) \neq-1$, then $\operatorname{Per}(f) \cap\{1,2\} \neq \emptyset$.

(d) If $g=1, E_{1}(A)=0$ and $E_{2}(A)=-1$, then there is no information on $\operatorname{Per}(f)$.

(e) If $g>1, E_{1}(A)=0, E_{2}(A)=1$ and $k$ is the smallest integer of the set $\{3,4, \ldots, 2 g\}$ such that $E_{k}(A) \neq 0$, then $f$ has a periodic point of period a divisor of $k$.

Unfortunately we cannot distinguish using the Lefschetz zeta function the orientation-preserving homeomorphisms from the orientation-reversing on $\mathbb{M}_{g, b}$ when $b>0$, see (2) and (3). They can be distinguished if $b=0$, because then they have different Lefschetz zeta functions, see again (2) and (3).

Proof of Theorem 2. Assume $b>0$. Let $f: \mathbb{M}_{g, b} \rightarrow \mathbb{M}_{g, b}$ be a homeomorphism, and let $A$ be the $(2 g+b-1) \times(2 g+b-1)$ integral matrix of the isomorphism $f_{* 1}: H_{1}(\mathbb{X}, \mathbb{Q}) \rightarrow H_{1}(\mathbb{X}, \mathbb{Q})$ induced by $f$ on the first homology group of $\mathbb{X}$. Then, combining the expressions (1), (2) with $b>0$, (3) with $b>0$ and (4) with $b \geq 0$ we obtain the following equalities

$$
\begin{aligned}
\sum_{n=1}^{\infty} \frac{L\left(f^{n}\right)}{n} t^{n} & =\log \left(Z_{f}(t)\right) \\
& =\log \left(\frac{\operatorname{det}(I-t A)}{1-t}\right) \\
& =\log \left(\frac{1-E_{1}(A) t+E_{2}(A) t^{2}-\ldots+(-1)^{m} E_{m}(A) t^{m}}{1-t}\right) \\
& =\log \left(1-E_{1}(A) t+E_{2}(A) t^{2}-\ldots\right)-\log (1-t) \\
& =\left(-E_{1}(A) t+\left(E_{2}(A)-\frac{E_{1}(A)^{2}}{2}\right) t^{2}-\ldots\right)-\left(-t-\frac{t^{2}}{2}-\ldots\right) \\
& =\left(1-E_{1}(A)\right) t+\left(\frac{1}{2}-\frac{E_{1}(A)^{2}}{2}+E_{2}(A)\right) t^{2}+O\left(t^{3}\right) .
\end{aligned}
$$

Here $m=2 g+b-1$ if $\mathbb{X}=\mathbb{M}_{g, b}$ with $b>0$, or $m=g+b-1$ if $\mathbb{X}=\mathbb{N}_{g, b}$ with $b \geq 0$. Therefore we have

$$
L(f)=1-E_{1}(A), \quad \text { and } \quad L\left(f^{2}\right)=1-E_{1}(A)^{2}+2 E_{2}(A) .
$$

Hence, if $E_{1}(A) \neq 1$ then $L(f) \neq 0$, and by Theorem 4 statement (a) follows.

If $E_{1}(A)=1$ and $E_{2}(A) \neq 0$, then $L\left(f^{2}\right)=2 E_{2}(A) \neq 0$, and again by Theorem 4 we get that $\operatorname{Per}(f) \cap\{1,2\} \neq \emptyset$. So statement (b) is proved. 
Assume now that $\mathbb{X}=\mathbb{M}_{g, b}$ with $b>0,2 g+b-1 \geq 3, E_{1}(A)=1, E_{2}(A)=0$ and $k$ is the smallest integer of the set $\{3,4, \ldots, 2 g+b-1\}$ such that $E_{k}(A) \neq 0$. Therefore

$$
\begin{aligned}
\sum_{n=1}^{\infty} \frac{L\left(f^{n}\right)}{n} t^{n} & =\log \left(\frac{1-t+(-1)^{k} E_{k}(A) t^{k}+\ldots+(-1)^{b-1} E_{2 g+b-1}(A) t^{2 g+b-1}}{1-t}\right) \\
& =\log \left(1+\frac{(-1)^{k} E_{k}(A) t^{k}+\ldots+(-1)^{b-1} E_{2 g+b-1}(A) t^{2 g+b-1}}{1-t}\right) \\
& =(-1)^{k} E_{k}(A) t^{k}+O\left(t^{k+1}\right) .
\end{aligned}
$$

Hence, $L(f)=\ldots=L\left(f^{k-1}\right)=0$ and $L\left(f^{k}\right)=(-1)^{k} k E_{k}(A) \neq 0$. So, from Theorem 4 , it follows the statement (c).

Suppose that $\mathbb{X}=\mathbb{N}_{g, b}$ with $b \geq 0, g+b-1 \geq 3, E_{1}(A)=1, E_{2}(A)=0$ and $k$ is the smallest integer of the set $\{3,4, \ldots, g+b-1\}$ such that $E_{k}(A) \neq 0$. Therefore

$$
\begin{aligned}
\sum_{n=1}^{\infty} \frac{L\left(f^{n}\right)}{n} t^{n} & =\log \left(\frac{1-t+(-1)^{k} E_{k}(A) t^{k}+\ldots+(-1)^{g+b-1} E_{g+b-1}(A) t^{g+b-1}}{1-t}\right) \\
& =\log \left(1+\frac{(-1)^{k} E_{k}(A) t^{k}+\ldots+(-1)^{g+b-1} E_{g+b-1}(A) t^{g+b-1}}{1-t}\right) \\
& =(-1)^{k} E_{k}(A) t^{k}+O\left(t^{k+1}\right) .
\end{aligned}
$$

Again $L(f)=\ldots=L\left(f^{k-1}\right)=0$ and $L\left(f^{k}\right)=(-1)^{k} k E_{k}(A) \neq 0$. Therefore, from Theorem 4 , it follows the statement (d).

\section{ACKNOWLEDGEMENTS}

The first author of this work was partially supported by MICINN/FEDER grant number MTM2011-22587, Junta de Comunidades de Castilla-La Mancha, grant number PEII09-0220-0222. The second author was partially supported by MICINN/FEDER grant number MTM2008-03437, AGAUR grant number 2009 SGR 410, ICREA Academia and FP7-PEOPLE-2012-IRSES numbers 316338 and 318999 . The third author was partially supported by Fundación Séneca de la Región de Murcia grant number 12001/PI/09.

\section{REFERENCES}

[1] R.F. Brown, The Lefschetz Fixed Point Theorem, Scott, Foresman and Company, Glenview, IL, 1971.

[2] J. Franks, Homology and Dynamical Systems, CBMS Regional Conf. Series, vol. 49, Amer. Math. Soc., Providence R.I., 1982.

[3] J. Franks and J. Llibre, Periods of surface homeomorphisms, Contemporary Mathematics 117 (1991), 63-77.

[4] F.B. Fuller, The existence of periodic points, Ann. of Math. 57, (1953), 229-230.

[5] B. Halpern, Fixed point for iterates, Pacific J. Math. 25 (1968), 255-275.

[6] J.R. Munkres, Elements of Algebraic Topology, Addison-Wesley, 1984.

[7] J.W. Vicks, Homology theory. An introduction to algebraic topology, Springer-Verlag, New York, 1994. Academic Press, New York, 1973. 
${ }^{1}$ Departamento de Matemática Aplicada y Estadística. Universidad Politécnica de Cartagena, Hospital de Marina, 30203-Cartagena, Región de Murcia, SPAIN.

E-mail address: juan.garcia@upct.es

${ }^{2}$ Departament de Matemàtiques. Universitat Autònoma de Barcelona, Bellaterra, 08193-Barcelona, Catalonia, Spain

E-mail address: jllibre@mat.uab.cat 